\title{
Signatures of the bonding-antibonding splitting in the $c$-axis infrared response of moderately underdoped bilayer and trilayer cuprate superconductors
}

\author{
B. P. P. Mallett,,${ }^{1,2,3,{ }^{*}}$ P. Marsik, ${ }^{3}$ D. Munzar, ${ }^{4}$ C. Bernhard,${ }^{3}$ and A. Dubroka ${ }^{4, \dagger}$ \\ ${ }^{1}$ The Photon Factory, Department of Physics, The University of Auckland, 38 Princes St, Auckland 1010, New Zealand \\ ${ }^{2}$ The MacDiarmid Institute for Advanced Materials and Nanotechnology, Victoria University of Wellington, PO Box 600, \\ Wellington 6140, New Zealand \\ ${ }^{3}$ University of Fribourg, Department of Physics and Fribourg Center for Nanomaterials, Chemin du Musée 3, CH-1700 Fribourg, Switzerland \\ ${ }^{4}$ Department of Condensed Matter Physics, Faculty of Science, and Central European Institute of Technology, Masaryk University, \\ Kotlářská 2, 61137 Brno, Czech Republic
}

(Received 16 September 2018; revised manuscript received 28 January 2019; published 22 February 2019)

\begin{abstract}
We report on results of our analysis of the $c$-axis infrared conductivity, $\sigma_{c}(\omega)$, of bilayer $\operatorname{LnBa}_{2} \mathrm{Cu}_{3} \mathrm{O}_{7-\delta}$ $(\mathrm{Ln}=\mathrm{La}, \mathrm{Nd}, \mathrm{Y})$ and trilayer $\mathrm{Bi}_{2} \mathrm{Sr}_{2} \mathrm{Ca}_{2} \mathrm{Cu}_{3} \mathrm{O}_{10+\delta}$ high- $T_{\mathrm{c}}$ superconductors. The analysis employs the multilayer model involving the conductivity of the bilayer or trilayer unit, $\sigma_{\mathrm{bl}}(\omega)$, and that of the spacing layers separating the latter units, $\sigma_{\text {int }}(\omega)$. For the $\mathrm{YBa}_{2} \mathrm{Cu}_{3} \mathrm{O}_{7-\delta}$ sample with concentration of holes $p=0.09$, our fitting of the data strongly suggests that at low temperatures, the conductivity $\sigma_{\mathrm{bl}}(\omega)$ possesses a pronounced and narrow Drude peak. For samples with $p \geqslant 0.115$ however, the fitting indicates that $\sigma_{\mathrm{bl}}(\omega)$ is, at low temperatures, dominated by a mode at a finite energy in the range from 30 to $60 \mathrm{meV}$. The properties of this resonance are in accord with those of a collective mode that appears in the spectra of $\sigma_{\mathrm{bl}}(\omega)$ calculated using a microscopic gauge-invariant theory of $\sigma_{c}(\omega)$ by J. Chaloupka and coworkers [Phys. Rev. B 79, 184513 (2009)]. The frequency and spectral weight of the latter mode are determined by the magnitude of the splitting between the bonding and the antibonding band of the bilayer or trilayer unit. Our results, in conjunction with the microscopic theory, thus demonstrate that in moderately underdoped bilayer and trilayer high- $T_{\mathrm{c}}$ cuprates the bilayer (or trilayer) splitting is already developed. The observed doping dependence is consistent with results from angular resolved photoemission spectroscopy.
\end{abstract}

DOI: 10.1103/PhysRevB.99.054513

\section{INTRODUCTION}

The $c$-axis infrared (IR) response of high- $T_{\mathrm{c}}$ cuprate superconductors contains a wealth of information that includes the temperature and energy scales of the pseudogap and the superconducting (SC) gap [1-8]. In materials with two $\mathrm{CuO}_{2}$ layers per unit cell (the so-called bilayer compounds), the $c$-axis response also reflects the electronic coupling within the pairs of closely spaced $\mathrm{CuO}_{2}$ layers. This coupling gives rise to a broad absorption peak in the spectra of the real part of the $c$-axis conductivity $\sigma_{c}(\omega)$, located in the frequency region between $350 \mathrm{~cm}^{-1}$ and $550 \mathrm{~cm}^{-1}[9,10]$. The peak, labeled as the transverse plasmon mode (TPM) in the following, onsets at a temperature $T^{\text {ons }}$ that, in underdoped cuprates, is higher than $T_{\mathrm{c}}$ but lower than the temperature scale, $T^{*}$, associated with the pseudogap [7]. The formation of the TPM is accompanied by pronounced changes of the spectral structures corresponding to some infrared-active phononsthe so-called phonon anomalies. These anomalies are well understood in terms of a coupling of the phonons to charge density oscillations between the closely spaced layers connected with the TPM. Despite the fact that the anomalies

\footnotetext{
*benjamin.mallett@gmail.com

†dubroka@physics.muni.cz
}

do not give evidence for a phonon-mediated mechanism of superconductivity, they represent a very sensitive probe of the low-energy charge excitations. This sensitivity was used to track the anomalous temperature dependence of the TPM in underdoped $\mathrm{YBa}_{2} \mathrm{Cu}_{3} \mathrm{O}_{7-\delta}(\mathrm{Y} 123)$, in particular its onset, far above $T_{\mathrm{c}}$ in strongly underdoped samples, that was interpreted in terms of superconducting or pairing fluctuations $[7,8]$. In addition, the TPM and the anomalies have gained considerable interest in the context of possible optical enhancement of superconductivity [11-13].

The TPM and the phonon anomalies in strongly underdoped samples have been modelled [7,14-16] successfully by an extension of the so-called multilayer model (MLM) originally proposed in Ref. [17]. Here, the bilayer cuprates are thought of as consisting of layers with distinctively different electronic properties. In particular, the layer limited by the closely spaced copper-oxygen layers-the so-called intrabilayer region-is much more conducting than the layer separating the bilayer blocks- the so-called interbilayer region. The conductivities of the former and of the latter will be denoted as $\sigma_{\mathrm{bl}}(\omega)$ and $\sigma_{\mathrm{int}}(\omega)$, respectively. The TPM can be interpreted in terms of resonant oscillations of the charge density between the closely spaced $\mathrm{CuO}_{2}$ layers, enabled by the difference between $\sigma_{\mathrm{bl}}(\omega)$ and $\sigma_{\text {int }}(\omega)$. These charge oscillations are infrared active and give rise to strong modifications of the local fields acting on the ions that participate in the phonon 
modes. This causes the phonon anomalies. It was indeed shown that the MLM provides an excellent description not only of the TPM but also of the phonon anomalies in the strongly underdoped cuprates $[7,14,15]$. In these studies it was assumed that the intrabilayer conductivity $\sigma_{\mathrm{bl}}(\omega)$ responsible for the TPM has its transverse frequency at zero, i.e., its spectra are similar to those of the bulk Drude response. However, it was suggested earlier [18] that the TPM could be related to the transitions between the bonding and antibonding bands of bilayer cuprates which can occur in the coherent limit relevant for higher dopings. The suggestion of Dordevic et al. [18] implicitly involves the idea that $\sigma_{\mathrm{bl}}(\omega)$ has a finite transverse frequency [i.e., $\sigma_{\mathrm{bl}, 1}(\omega)$ displays a maximum at a finite frequency]. More recently, detailed microscopic calculations of the $c$-axis response were performed by some of us using a nonperturbative approach involving the bilayer split bands [19]. These calculations, which are relevant for the limit of two well developed bands, showed that the transverse frequency of $\sigma_{\mathrm{bl}}(\omega)$ indeed does not occur at zero but at a finite frequency. Early angular resolved photoemission spectroscopy (ARPES) experiments had failed to resolve a bilayer splitting of the conduction band and it was not until overdoped $\mathrm{Bi}_{2} \mathrm{Sr}_{2} \mathrm{CaCu}_{2} \mathrm{O}_{8-\delta}$ (Bi2212) samples were measured that the bonding and antibonding bands were individually resolved [20-22]. More recently, splitting between the two bands was observed by ARPES in optimally doped $\mathrm{Bi}_{2} \mathrm{Sr}_{2} \mathrm{Ca}_{2} \mathrm{Cu}_{3} \mathrm{O}_{10-\delta}$ (Bi2223) [23] and in $\mathrm{YBa}_{2} \mathrm{Cu}_{3} \mathrm{O}_{7-\delta}\left(\mathrm{Y}_{123}\right.$ ) [24-26].

Inspired by these findings, we seek to discern the effect of the conduction band splitting in the $c$-axis response of several moderately underdoped cuprates. We study $\mathrm{LnBa}_{2} \mathrm{Cu}_{3} \mathrm{O}_{7-\delta}$ $(\mathrm{Ln}=\mathrm{Y}, \mathrm{Nd}, \mathrm{La})$ with hole doping from $p \approx 0.1$ to 0.16 and the Bi2223 trilayer cuprate superconductor. We use the MLM scheme to fit our data and allow the local intrabilayer conductivity $\sigma_{\mathrm{bl}}(\omega)$ to be either centered at zero or at a finite frequency, i.e., to model negligible or significant bondingantibonding splitting, respectively. We show here that the model with the finite transverse frequency of $\sigma_{\mathrm{bl}}(\omega)$ indeed yields significantly better fits to the data for moderately underdoped samples $(p \geqslant 0.115)$. This provides evidence for bonding-antibonding splitting of the conduction band in the bulk for bi- and trilayer cuprates.

\section{METHODS}

\section{A. Samples and measurements}

The high quality twinned $R \mathrm{Ba}_{2} \mathrm{Cu}_{3} \mathrm{O}_{7-\delta}(R=\mathrm{La}, \mathrm{Nd}$, and $\mathrm{Y})$ crystals were flux grown in Y-stabilized zirconium crucibles. For $R=\mathrm{Nd}$, the crystals were grown under reduced oxygen atmosphere to avoid spurious substitution of the $\mathrm{Nd}$ ion onto the $\mathrm{Ba}$ site [27]. The crystals were annealed, while covered in $\mathrm{YBa}_{2} \mathrm{Cu}_{3} \mathrm{O}_{7-\delta}$ (Y123) powder to protect their surfaces, in a pure oxygen atmosphere at various temperatures in order to alter $\delta$ and hence the electronic doping of the $\mathrm{CuO}_{2}$ layers. When necessary the crystals were mechanically polished to optical grade using diamond powder paste, however, virgin surfaces were used where possible. The typical $c$-axis dimension of our Y123 single crystals was 0.5 to $1 \mathrm{~mm}$. Since the $c$-axis response is insulatorlike, the diffraction effects are much less pronounced than for the metallic in-plane response.
For the $c$-axis response of these Y123 crystals, diffraction effects set in gradually with decreasing wave number and become noticeable below about $200 \mathrm{~cm}^{-1}$ where they mainly cause a decrease of the spectral weight of the phonons. The $c$-axis dimensions of the $\mathrm{Nd} 123$ and La123 crystals are about $2 \mathrm{~mm}$ and thus the diffraction sets in at even lower frequencies than for the Y123 crystals.

The $c$-axis IR response was measured using broadband ellipsometry. Ellipsometry is a self-normalizing optical technique to directly measure the complex conductivity, $\sigma(\omega)=$ $-\mathrm{i} \varepsilon_{0} \omega[\epsilon(\omega)-1]$, without the need for a Kramers-Kronig analysis [28]. We used an in-house built ellipsometer attached to a Bruker fast-Fourier spectrometer at the infrared beam line of the ANKA synchrotron at the Karlsruhe Institute for Technology (KIT) to measure in the far-IR range from $70 \mathrm{~cm}^{-1}$ to $700 \mathrm{~cm}^{-1}$, see Ref. [29], and a similar laboratorybased setup at University of Fribourg covering the far-IR to mid-IR range, $100 \mathrm{~cm}^{-1}$ to $4500 \mathrm{~cm}^{-1}$. For these ellipsometry measurements we used a rotating analyzer configuration and anisotropy corrections were performed where necessary using standard numerical procedures [28,29].

\section{B. Multilayer model fitting}

We have analyzed our data with the MLM [14] whose details have been described previously and we use here the same structure of the interaction between the phonons and the electronic degrees of freedom as in Refs. [16,30]. For clarity however, we repeat a few key expressions below. The $c$-axis response in the optical limit is written as a sum of volume-averaged (microscopic) current densities, $\left\langle j_{k}(\omega)\right\rangle$ :

$$
\epsilon(\omega)=\epsilon_{\infty}+\frac{\mathrm{i}}{\epsilon_{0} \omega} \frac{\sum_{k}\left\langle j_{k}(\omega)\right\rangle}{E(\omega)} .
$$

Here, $\epsilon_{\infty}$ is the high frequency dielectric constant (a fitted parameter) and $E(\omega)$ the total electric field along the $c$ axis. Next, the expression above is expanded as follows:

$$
\begin{aligned}
\epsilon(\omega)= & \epsilon_{\infty} \\
& +\frac{d_{\mathrm{bl}}}{d_{\mathrm{bl}}+d_{\mathrm{int}}} \frac{\mathrm{i}}{\epsilon_{0} \omega} \frac{j_{\mathrm{bl}}(\omega)}{E(\omega)}+\frac{d_{\mathrm{int}}}{d_{\mathrm{bl}}+d_{\mathrm{int}}} \frac{\mathrm{i}}{\epsilon_{0} \omega} \frac{j_{\mathrm{int}}(\omega)}{E(\omega)} \\
& +\chi_{\mathrm{int}}^{Q}(\omega) \frac{E_{\mathrm{int}}^{\mathrm{loc}}(\omega)}{E(\omega)}+\chi_{\mathrm{ocp}}^{Q}(\omega) \frac{E_{\mathrm{ocp}}^{\mathrm{loc}}(\omega)}{E(\omega)}+\chi_{\mathrm{Lor}}^{Q}(\omega) .
\end{aligned}
$$

The second and the third terms represent the contributions of the intrabilayer and interbilayer current densities, denoted by "bl" and "int," respectively. The fourth, fifth, and sixth terms describe the contributions of phonons driven by the local fields, $E_{\text {int }}^{\text {loc }}(\omega)$, of the interbilayer region, $E_{\text {ocp }}^{\text {loc }}(\omega)$ acting on the (outer) $\mathrm{CuO}_{2}$ layers and by the average electric field, $E(\omega)$, respectively. The last term includes standard Lorentz phonon lineshapes corresponding to noninteracting (mean field) phonons. The intrabilayer and interbilayer current densities are given by the corresponding averaged local fields and local conductivities:

$$
\begin{aligned}
& j_{\mathrm{bl}}(\omega)=\sigma_{\mathrm{bl}}(\omega) E_{\mathrm{bl}}(\omega), \\
& j_{\mathrm{int}}(\omega)=\sigma_{\mathrm{int}}(\omega) E_{\mathrm{int}}(\omega) .
\end{aligned}
$$


The conductivities are represented in terms of the corresponding polarizabilities, $\chi_{\mathrm{bl}}(\omega)$ and $\chi_{\mathrm{int}}(\omega)$, as $\sigma_{\mathrm{bl}}(\omega)=$ $-\mathrm{i} \omega \epsilon_{0} \chi_{\mathrm{bl}}(\omega)$ and $\sigma_{\mathrm{int}}(\omega)=-\mathrm{i} \omega \epsilon_{0} \chi_{\mathrm{int}}(\omega)$. Drude-Lorentz terms are used to describe components of all the polarizabilities involved $\left(\chi_{\mathrm{bl}}, \chi_{\mathrm{int}}, \chi_{\mathrm{int}}^{Q}, \chi_{\mathrm{ocp}}^{Q}\right.$, and $\left.\chi_{\text {Loc }}^{Q}\right)$, each of them is expressed as

$$
\chi(\omega)=\sum_{m} \frac{\omega_{\mathrm{pl}, m}^{2}}{\omega_{0, m}^{2}-\omega^{2}-\mathrm{i} \omega \gamma_{m}}
$$

with the resonant frequency $\omega_{0, m}$ being either finite or zero for a Lorentz or Drude term, respectively. Detailed formulas for the averaged local fields [e.g., $\left.E_{\mathrm{bl}}(\omega)\right]$ and the local fields acting on the ions [e.g., $\left.E_{\text {ocp }}^{\text {loc }}(\omega)\right]$ are given in Ref. [30].

In summary, the MLM represents a set of self-consistent equations describing the coupling between the lattice vibrations and the local current densities via electrostatic interaction. The equations can be derived starting from a fully microscopic theory involving Green's function [31]. As a result, phonon anomalies, such as an asymmetrical line shape or atypical temperature dependence, can be accounted for as a consequence of changes in $\sigma_{\mathrm{bl}}(\omega)$ and/or $\sigma_{\mathrm{int}}(\omega)$.

\section{DATA AND ANALYSIS}

\section{A. $\mathrm{YBa}_{2} \mathrm{Cu}_{3} \mathrm{O}_{6.65}$ with $p=0.115$}

To demonstrate how a bilayer splitting may be discerned from IR spectroscopy data, we focus first on an underdoped Y123 sample with $T_{\mathrm{c}}=75 \mathrm{~K}$ and $p=0.115$. Figure 1(a) shows the real and imaginary parts of the $c$-axis IR conductivity measured at $120 \mathrm{~K}$ (red lines) and $10 \mathrm{~K}$ (cyan lines). The spectra display six IR active phonon modes and an electronic background. The largest changes with cooling to $10 \mathrm{~K}$ occur between $250-700 \mathrm{~cm}^{-1}$ where the broad band centered at 480 $\mathrm{cm}^{-1}$ - the TPM-emerges and the phonon peaks at 190, 280, 320,560 , and $630 \mathrm{~cm}^{-1}$ exhibit sizable spectral changes. The latter effects are the well known phonon anomalies that were recognized and extensively studied earlier $[4,5,7,14,32]$.

In order to quantitatively analyze the data, we use the MLM with the same assumptions as in our earlier studies, i.e., the phonons at 280,560, $630 \mathrm{~cm}^{-1}$ that involve vibrations of the apical and chain oxygen are driven by the local field $E_{\text {int }}^{\text {loc }}(\omega)$ and the phonon at $320 \mathrm{~cm}^{-1}$ that involves vibrations of the planar oxygens is driven by the local field $E_{\text {ocp }}^{\text {loc }}(\omega)$. The only difference as discussed below is that we show how the model spectra fit the data in two different cases: (a) if the resonance frequency of $\chi_{\mathrm{bl}}(\omega)$ is fixed at zero and (b) if it is allowed to be finite. The two low frequency phonons at 155 and $190 \mathrm{~cm}^{-1}$ are treated as mean field phonons whose parameter values are fitted at each temperature. Note that the $190 \mathrm{~cm}^{-1}$ phonon peak, although its spectral weight is small exhibits sizable anomaly as well: The spectral weight increases significantly when going from the normal to the superconducting state. This anomaly is related to the temperature dependence of the intrabilayer local field and to the complex polarization

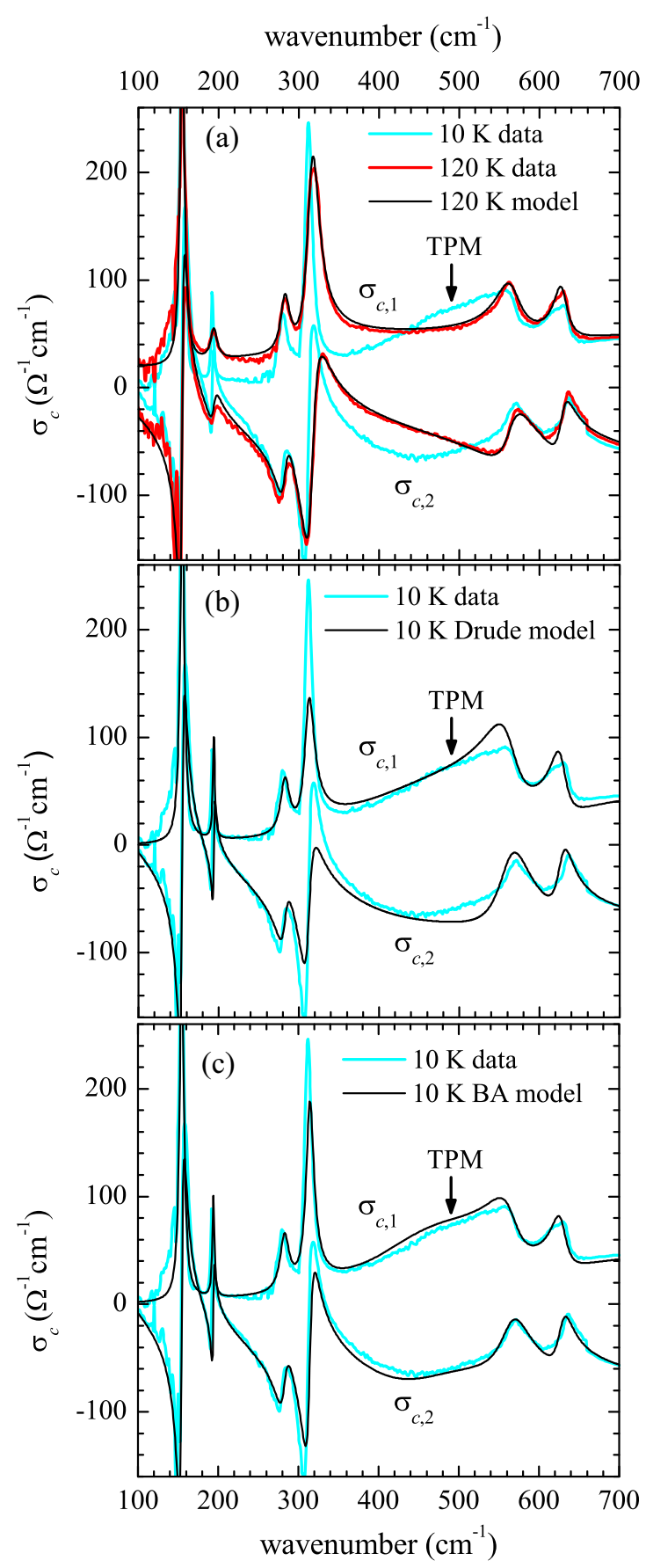

FIG. 1. The real and imaginary parts of the $c$-axis conductivity, $\sigma_{c, 1}(\omega)$ and $\sigma_{c, 2}(\omega)$, for the Y123 sample with $p=0.115$ at $120 \mathrm{~K}$ (red lines) and $10 \mathrm{~K}$ (cyan lines). The panels show also the fitted spectra obtained using the MLM (black lines) for (a) $120 \mathrm{~K}$, (b) for $10 \mathrm{~K}$ with $\sigma_{\mathrm{bl}}(\omega)$ described using the Drude model, and (c) for $10 \mathrm{~K}$ with $\sigma_{\mathrm{bl}}(\omega)$ described using the BA model. The arrow shows the center frequency of the transverse plasma mode (TPM).

diagram of the phonon [16]. ${ }^{1}$ Its description is beyond the scope of this paper.

\footnotetext{
${ }^{1}$ The eigenvector of the $190 \mathrm{~cm}^{-1}$ phonon in Y123 involves mainly the in-phase motion of yttrium and planar oxygen ions, see Ref. [45]. However, since the effective charge of oxygen is negative and that
} 
A key feature of our fitting is that the values of the parameters of the phonons driven by the local fields (the four phonons above $250 \mathrm{~cm}^{-1}$ in the case of Y-123) are obtained by fitting the data above $T_{\mathrm{c}}$ (usually at $120 \mathrm{~K}$ ) and are then kept fixed when fitting the data sets corresponding to lower temperatures. This leaves only variations in $\sigma_{\mathrm{bl}}(\omega)$ [and $\left.\sigma_{\text {int }}(\omega)\right]$ to describe the low temperature electronic response, the TPM, and the phonon anomalies. Consequently, the spectra of $\sigma_{\mathrm{bl}}(\omega)$ and $\sigma_{\text {int }}(\omega)$ are well determined. Both real and imaginary parts of the conductivity, $\sigma_{c, 1}(\omega)$ and $\sigma_{c, 2}(\omega)$, are used for fitting up to $4000 \mathrm{~cm}^{-1}$ (except for the Bi-based cuprates for which we only have the data up to $700 \mathrm{~cm}^{-1}$ ). This reduces ambiguities in the determination of the local conductivities.

The black lines in Fig. 1(a) show the fit of the MLM to the data for $120 \mathrm{~K}$. At this doping, the values of the oscillator parameters of the $320 \mathrm{~cm}^{-1}$ phonon are $\omega_{\mathrm{pl}}=460 \mathrm{~cm}^{-1}$ and $\omega_{0}=410 \mathrm{~cm}^{-1}$. The values of the two frequencies are very similar to those of a Y123 sample with $T_{\mathrm{c}}=58 \mathrm{~K}$ and $p=$ 0.09 obtained using the MLM model recently [7]. Values of all the parameters are tabulated in Table I.

When applying the MLM to the low temperature data, we find that it is the form of the intrabilayer conductivity, $\sigma_{\mathrm{bl}}(\omega)$, rather than the interbilayer conductivity, $\sigma_{\text {int }}(\omega)$, that determines the shape of the TPM. We first assume that the main contribution to $\sigma_{\mathrm{bl}}(\omega)$ in the far-IR frequency range is given by a Drude peak with $\gamma=0$. Previously, such a model was successfully used in fitting the data of the strongly underdoped Y123 sample with $T_{\mathrm{c}}=58 \mathrm{~K}[7,14]$. The model with this assumption will be denoted as the "Drude model" and the corresponding fits are shown in Fig. 1(b) using the black lines. Although the Drude model based fits for our $p=0.115 \mathrm{~K}$ sample capture the main trends in the spectra, there are significant deviations: (i) the spectral weight of the phonons at 560 and $630 \mathrm{~cm}^{-1}$ is overestimated, (ii) the TPM feature does not have a convex (bellylike) shape but rather a concave shape, and finally (iii) the spectral weight of the $320 \mathrm{~cm}^{-1}$ phonon is too low.

Next, we relax our earlier assumption and allow the main contribution to the intrabilayer conductivity $\sigma_{\mathrm{bl}}(\omega)$ in the farIR range to be centered at a finite frequency. We have labeled this approach as the "BA model," motivated by the similarity between the corresponding mode of the microscopic theory of Ref. [19] ("BA mode") and the Bogolyubov-Anderson mode of a single layer superconductor. Figure 1(c) and, over an extended spectral range, Fig. 2(a) show the corresponding model spectra where the resonance of $\chi_{\mathrm{bl}}(\omega)$ is centered at about $260 \mathrm{~cm}^{-1}$ and the Drude peak with $\gamma=0$ is absent, as shown in Fig. 2(b) which displays the local conductivities. It is clear that this model fits the data much better in all three aspects

of yttrium positive, the corresponding polarization pattern of the eigenvector has an out-of-phase character. It was shown that an out-of-phase resonance of ions in the outer $\mathrm{CuO}_{2}$ layers and in the intrabilayer region is strongly enhanced in the superconducting state if it is located at lower frequency than that of the TPM, see Fig. 8(a) in Ref. [16]. We believe that this is the main cause for the observed strong enhancement of the phonon at $190 \mathrm{~cm}^{-1}$ in $\mathrm{Y} 123$. The phonon at $400 \mathrm{~cm}^{-1}$ in Bi2223 is anomalous for similar reasons, see Sec. C.

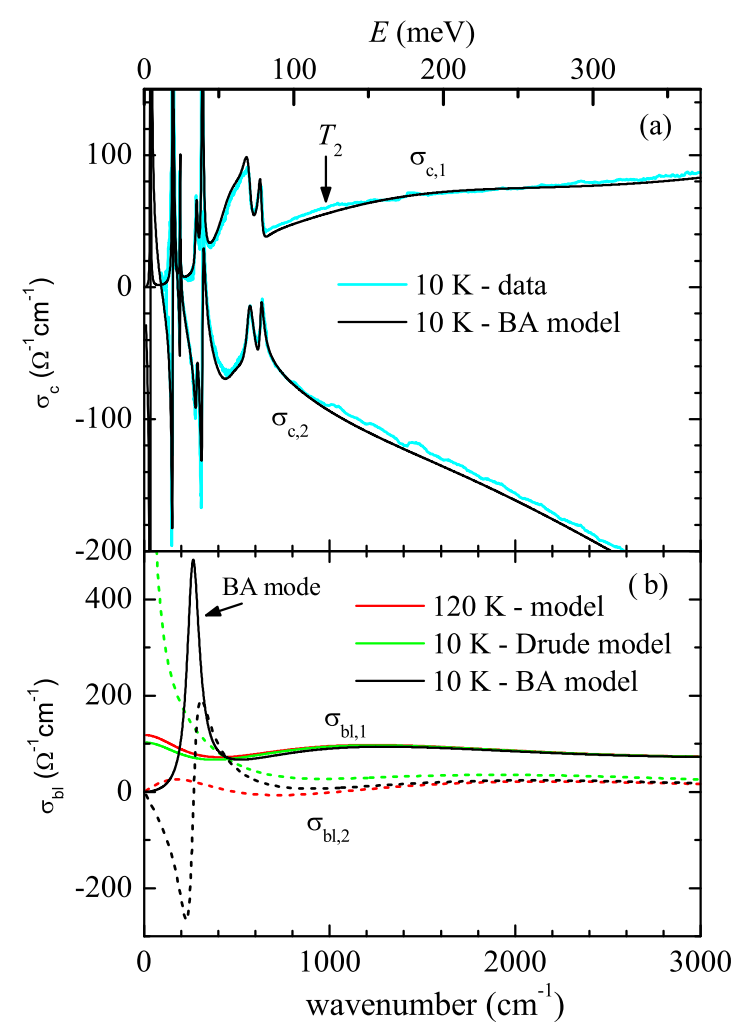

FIG. 2. Panel (a) shows the $10 \mathrm{~K}$ data for the Y123 sample with $p \approx 0.115$ (cyan lines) and the MLM model based fit (black lines) over an extended spectral range. (b) The real (solid lines) and imaginary (dotted lines) parts of the local intrabilayer conductivity $\sigma_{\mathrm{bl}}(\omega)$ involved in the fits shown in Fig. 1 and in panel (a).

for which the Drude model failed. Particularly the bellylike shape of the TPM feature and the phonon anomalies of the 560 and $630 \mathrm{~cm}^{-1}$ phonons are significantly better reproduced. We note however that a weak Drude term in $\sigma_{\mathrm{bl}}(\omega)$, i.e., a weak SC delta function in the real part of $\sigma_{\mathrm{bl}}(\omega)$, with a spectral weight much smaller than that of the finite frequency mode, would not be inconsistent with the present fit in the region of the TPM. Consequently we do not claim that the SC delta function is completely absent, we just claim that the main cause for the observed TPM and the phonon anomalies at this doping is the finite frequency mode and not the SC delta function. Actually, a weak SC delta function, with the plasma frequency comparable to that of $\sigma_{\text {int }}(\omega)$ (see Table I), must be present also in $\sigma_{\mathrm{bl}}(\omega)$ for the whole multilayer structure to exhibit a dc superconducting response.

Next, we discuss in more detail the spectra of $\sigma_{\mathrm{bl}}(\omega)$ obtained from the fits, which are shown in Fig. 2(b). First, we can see that the high frequency part above $1000 \mathrm{~cm}^{-1}$ is essentially temperature and model independent with the major changes occurring at lower frequencies. At $120 \mathrm{~K}$, the response is essentially incoherent with a broad Drude term with the plasma frequency $\omega_{\mathrm{pl}}=1400 \mathrm{~cm}^{-1}$ and the broadening parameter $\gamma=280 \mathrm{~cm}^{-1}$. Considering the width of the Drude term, the question as to whether the resonance is at zero or at small finite frequency cannot be answered.

The solid green line in Fig. 2(b) represents the spectrum of $\sigma_{\mathrm{bl}, 1}(\omega)$ corresponding to the Drude model based fit for 
TABLE I. Values of the fitting parameters entering the model formulas: frequencies $\left(\omega_{0}\right)$, plasma frequencies $\left(\omega_{\mathrm{pl}}\right)$, and broadening parameters $(\gamma)$ of the oscillators obtained by fitting the high temperature $(100-160 \mathrm{~K})$ and the $10 \mathrm{~K}$ data of Ln123 crystals. All values of phonon parameters have been obtained by fitting the high temperature data and fixed for $10 \mathrm{~K}$ fitting except for some phonon parameters of Y123 with $p=0.09$ that were allowed to vary to accommodate for the large temperature difference analyzed in Ref. [7].

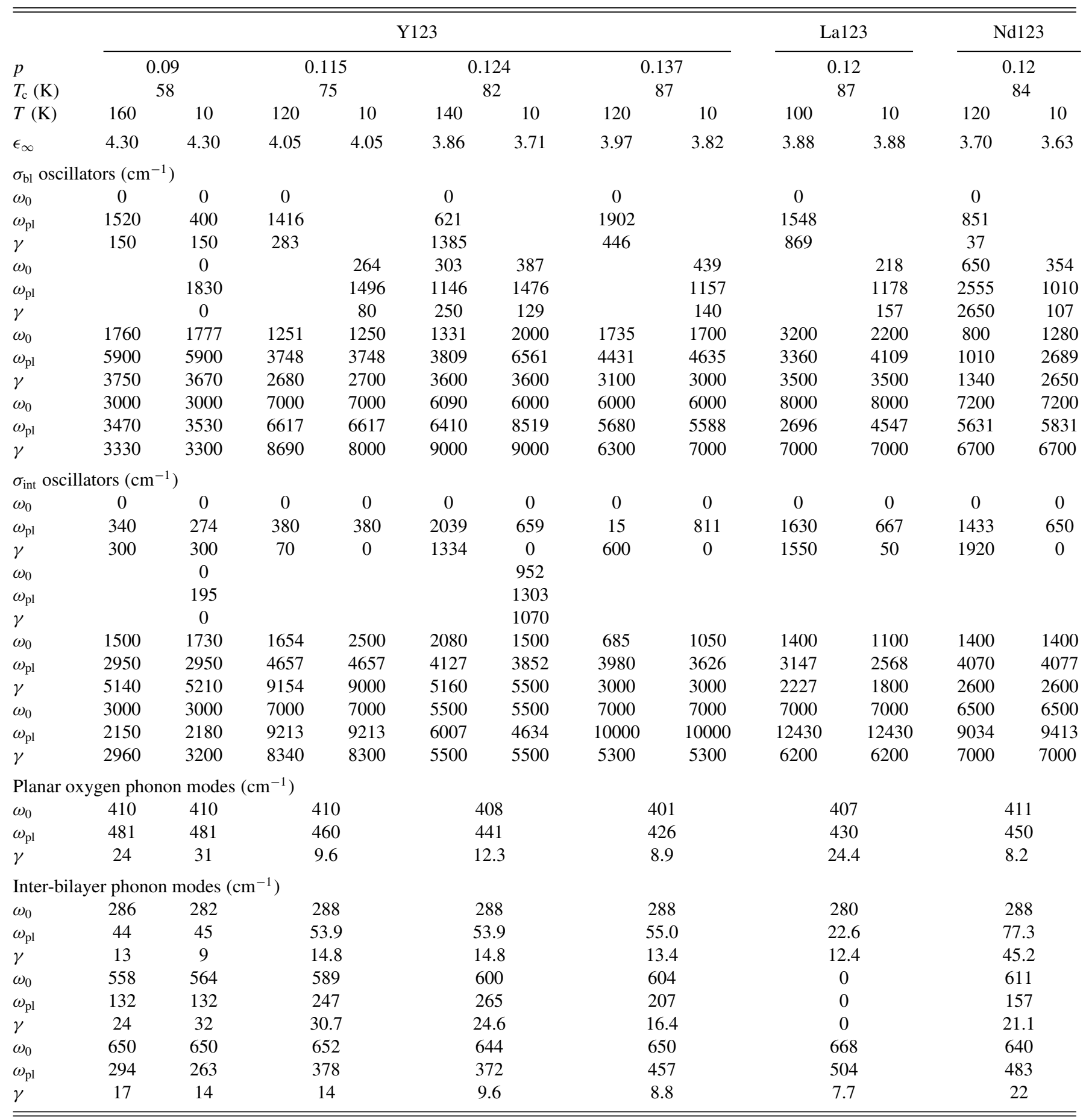

$10 \mathrm{~K}$. It looks very similar to the one of $T=120 \mathrm{~K}$, however it has a delta function at zero frequency with quite a significant plasma frequency of $\omega_{\mathrm{pl}}=1400 \mathrm{~cm}^{-1}$ giving rise to the low-frequency upturn in $\sigma_{\mathrm{bl}, 2}(\omega)$. The solid black line represents the spectrum corresponding to the BA model at $10 \mathrm{~K}$. It exhibits a sharp mode centered at $260 \mathrm{~cm}^{-1}$ with a plasma frequency of about $1500 \mathrm{~cm}^{-1}$, to be labeled as BA mode in the following. Note that the frequency of the BA mode is significantly lower than $470 \mathrm{~cm}^{-1}$, the frequency of the TPM. This is because the TPM occurs close to the screened longitudinal frequency of the intrabilayer response that is determined by both the transverse frequency and the spectral weight of the BA mode, for details see Appendix. 


\section{B. Interpretation of the BA mode}

The properties of the peak in $\sigma_{\mathrm{bl}, 1}(\omega)$ discussed above are well comparable to those of the BA mode occurring in the spectra of $\sigma_{\mathrm{bl}}(\omega)$ reported in Ref. [19], obtained using a microscopic gauge-invariant theory involving the bilayer-split (bonding and antibonding) bands; see Figs. 10(d) and 14(a) of Ref. [19], in particular the spectra corresponding to $t_{\perp}=45$ $\mathrm{meV}$ in Fig. 10(d) and $t_{\perp}=30 \mathrm{meV}$ in Fig. 14(a). Here $t_{\perp}$ is the intrabilayer hopping parameter.

\section{Bogolyubov-Anderson mode}

The nature of the BA mode of Ref. [19] is similar to that of the Bogolyubov-Anderson mode that participates in the longitudinal response of a homogeneous superconductor. We recall that in homogeneous superconductors a longitudinal electromagnetic field excites the Bogolyubov-Anderson mode involving density fluctuations of the electron system, associated with a modulation of the phase of the order parameter [33-35]. The energy of the Bogolyubov-Anderson mode is proportional to $v_{F}|\boldsymbol{q}|$, where $v_{F}$ is the Fermi velocity and $\boldsymbol{q}$ the wave vector. So far we did not consider the Coulomb interaction between the carriers, that will shift the mode towards higher frequencies. In a single-layer superconductor (one $\mathrm{CuO}_{2}$ plane per unit cell), a longitudinal electromagnetic field with $\boldsymbol{E} \| c$ would induce a Bogolyubov-Anderson-like mode with energy proportional to the Fermi velocity along the $c$ axis. Again the Coulomb interaction shifts the mode towards higher frequencies. In the long-wavelength limit we obtain the $c$-axis plasma mode.

\section{Relation between the BA mode and the Bogolyubov Anderson mode}

In the present case of the IR response of a bilayer superconductor the situation is more complicated. The electromagnetic wave is transverse with $\boldsymbol{q} \perp c$. Nevertheless, it induces a charge density that is modulated along the $c$ axis. For small values of $t_{\perp}$ the modulation is accompanied by oscillations of the relative phase of the two planes, see Fig. 4(b) of Ref. [19]. The pattern is analogous to the one associated with the Bogolyubov-Anderson mode of a single-layer superconductor with $\boldsymbol{q} \| c,|\boldsymbol{q}|=\pi / d$ shown in Fig. 4(a) of Ref. [19]. The analogy allows us to interpret the BA mode of $\sigma_{\mathrm{bl}}$ as an analog of the Bogolyubov-Anderson mode. This point of view can be substantiated by comparing the equations describing the modes as detailed in Ref. [19]. Note that the long-wavelength in-plane modulation of the electromagnetic wave has qualitatively no impact on the mode. In the total conductivity the BA mode is shifted to higher energies by the interlayer Coulomb interaction. The resulting structure corresponding as we believe to the TPM is labeled as $T_{1}$ in Ref. [19].

\section{Relation between the energy of the BA mode and the bonding-antibonding splitting}

Note that the energy of the BA mode is proportional to $t_{\perp}$, i.e., to the bilayer splitting (the unrenormalized magnitude of the band splitting is $2 t_{\perp}$ ). It has been argued by Hirata $e t$ al. [36] that the strong temperature dependence of the TPM mode in multilayer Hg-based cuprates below $T_{c}$ indicates that the mode is rather due to the plasma of the superconducting pairs than to an interband transition. We agree that the temperature dependence suggests that superconductivity is a prerequisite for the formation of the TPM. But in addition there is, we believe, a relation to the bonding-antibonding splitting that we explain below. According to the model of Ref. [19] the normal state spectra of $\sigma_{\mathrm{bl}, 1}(\omega)$ display a very broad band that can be roughly interpreted as an overdamped interband bondingantibonding transition, see Fig. 10(b) of Ref. [19]. In the total conductivity the resulting structure is even broader, see Fig. 10(e) of Ref. [19]. In the superconducting state, however, the band in $\sigma_{\mathrm{bl}, 1}(\omega)$ becomes fairly narrow, see Fig.10(d) of Ref. [19]. A sharp band shifted to higher energies appears then in the spectra of the total conductivity, see Fig. 10(f) of Ref. [19]. The important questions are: (a) Why is the energy of the band in the superconducting state unrelated to the superconducting energy gap $\Delta$ ? (b) Why is the band so narrow?

Regarding (a): the infrared radiation excites quasiparticles. A typical final state-in the absence of interactions-would involve a Bogoljubov quasiparticle of the bonding band (energy $\left.E_{b}=\sqrt{\Delta_{b}^{2}(\boldsymbol{k})+\epsilon_{b}^{2}(\boldsymbol{k})}\right)$ and a Bogoljubov quasiparticle of the antibonding band (energy $\left.E_{a}=\sqrt{\Delta_{a}^{2}(\boldsymbol{k})+\epsilon_{a}^{2}(\boldsymbol{k})}\right)$. Here $\Delta_{b}$ and $\Delta_{a}$ are the superconducting gaps of the two bands and $\epsilon_{b}$ and $\epsilon_{a}$ the corresponding dispersions. The total energy of the excited state would be $E_{b}(\boldsymbol{k})+E_{a}(\boldsymbol{k})$. The excited quasiparticles however interact and form a bound state (this is similar to the formation of an exciton below the band gap of a semiconductor), whose energy is determined by $t_{\perp}$ rather than by $\Delta$.

Regarding (b): The band is so narrow because the quasiparticle background is strongly suppressed by the formation of the superconducting gap. As the band moves into the quasiparticle continuum above $2 \Delta$, it becomes fairly broad, see Figs. 10(d) and 10(f) of Ref. [19].

These facts explain the rapid development below $T_{c}$ of the band inside the superconducting gap. Note that for small values of the bilayer splitting, the nature of the BA mode is very similar to that of the bilayer plasmon of the phenomenological Josephson superlattice model used in many earlier studies.

\section{Pair breaking bonding-antibonding transition}

We have stressed that the BA mode and the TPM cannot be simply interpreted in terms of a transition between the bonding and the antibonding bands. Such a transition (a pairbreaking bonding-antibonding transition) is predicted to occur somewhat above the superconducting gap and indeed a second superconductivity-induced mode appears in the data at higher frequencies [19]. For our Y123 sample with $p=0.115$, the latter mode occurs at about $120 \mathrm{meV}$ and it is denoted as $T_{2}$ in Fig. 2(a). Next we address the nature of this second mode. In our discussion of the energy of the BA mode above, we have mentioned final states with energy $E_{b}(\boldsymbol{k})+E_{a}(\boldsymbol{k})$. In the expressions for $\sigma_{\mathrm{bl}}$ they are coupled and yield a bound state (excitonlike) inside the superconducting gap. In the expression for $\sigma_{\text {int }}$ they are not coupled because there are, within the model, no interactions between different bilayers. 

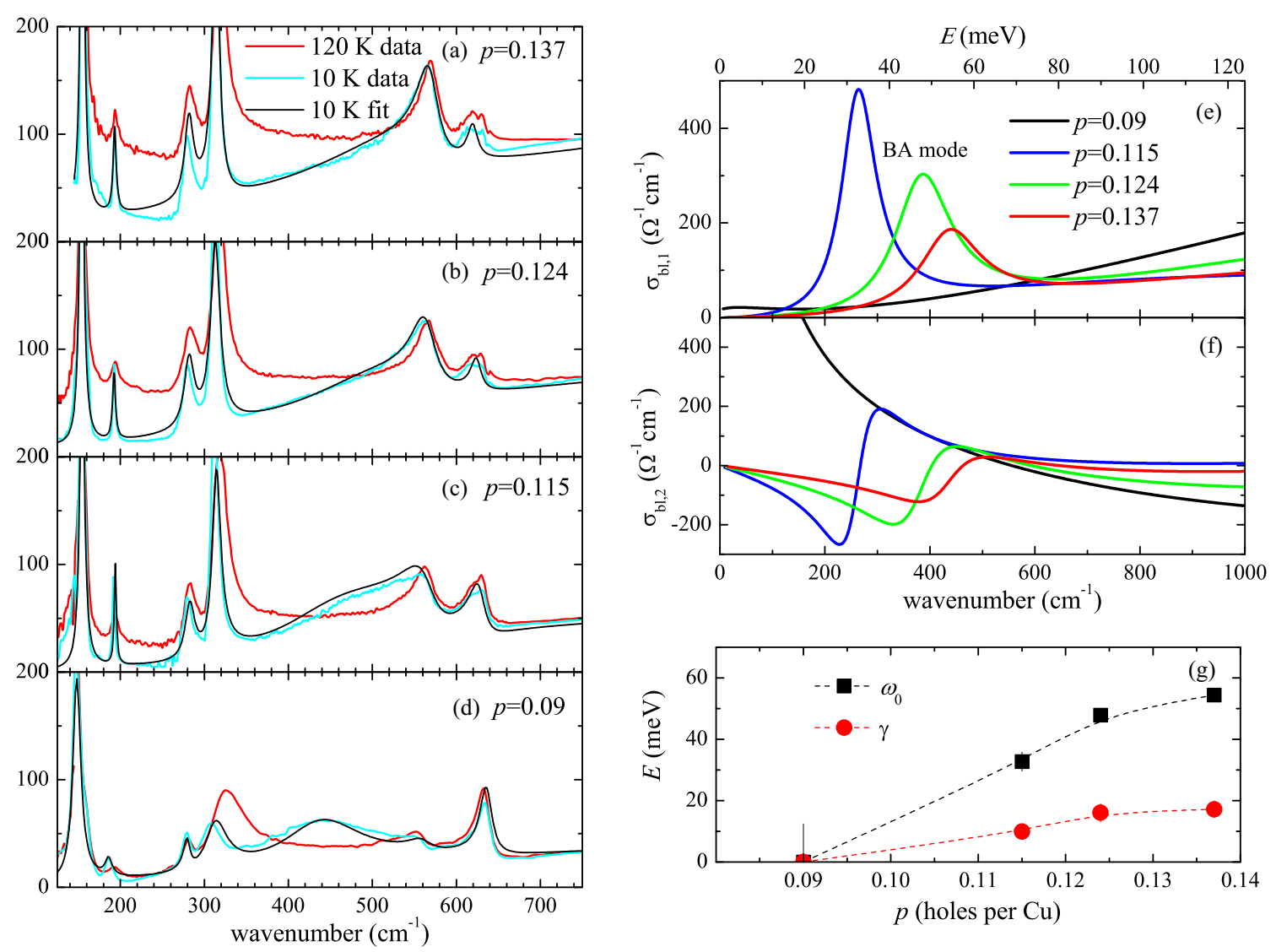

FIG. 3. Doping dependence of the BA mode in Y123. Panels (a)-(d) show the $T=10 \mathrm{~K}$ spectra of $\sigma_{c, 1}(\omega)$ (cyan lines) and the $120 \mathrm{~K}$ spectra (red lines) for Y123 with various values of the doping $p$. The black lines are fits of the $10 \mathrm{~K}$ data obtained using the MLM. Panels (e) and (f) show the real and imaginary parts of $\sigma_{\mathrm{bl}}(\omega)$, respectively, inferred from the MLM fitting for $10 \mathrm{~K}$. Panel (g) shows the doping evolution of the BA mode's frequency, $\omega_{0}$, and its broadening parameter $\gamma$. The lines are guides to the eye.

These final states give rise to a structure somewhat above $2 \Delta$, see Fig. 13 of Ref. [19]. The energy is above $2 \Delta$ because of the factors $\epsilon_{b}(\boldsymbol{k})$ and $\epsilon_{a}(\boldsymbol{k})$ in $E_{b}(\boldsymbol{k})$ and $E_{a}(\boldsymbol{k})$ that cannot be simultaneously zero.

\section{Doping and intralayer distance dependence}

Here we address the doping and intrabilayer distance dependencies of the BA mode inferred from our MLM fitting and compare it with expectations based on theory and ARPES measurements. Figures 3(a)-3(d) shows $\sigma_{c, 1}(\omega)$ data for Y123 with $p$ ranging from 0.137 to 0.09 , along with the corresponding fits obtained using the MLM. We use the same version of the MLM here for all doping values - we only vary the values of the fitting parameters that are listed in Table I.

In the case of the strongly underdoped sample with $p=$ 0.09 , the best fit is obtained using the Drude model, see Fig. 3(d). The model with the frequency of the BA mode in the range $150-300 \mathrm{~cm}^{-1}$ led to worse agreement with the data (not shown). Note that based on the data it cannot be decided whether the resonance of $\sigma_{\mathrm{bl}}(\omega)$ is located at $\omega=0$ or at a finite $\omega$ smaller than about $100 \mathrm{~cm}^{-1}$ because the differences between the fits are not significant.

With increasing doping, the TPM moves to higher energies where it eventually merges with the continuum for $p \approx 0.15$ in Y123 [30]. Meanwhile, the associated phonon anomalies become progressively weaker. As the TPM and the anomalies become less pronounced, the results of the MLM analysis become less reliable. The highest doping for which we were able to perform a reliable MLM based data analysis is $p=0.137$ for Y123 with $T_{\mathrm{c}}=87 \mathrm{~K}$ [see Fig. 3(a)] and $p \approx 0.145$ for Nd123 with $T_{\mathrm{c}}=94 \mathrm{~K}$ (the effects of the substitution of $\mathrm{Nd}$ for $\mathrm{Y}$ are addressed below). Figures 3(e) and 3(f) display the inferred real and imaginary parts of $\sigma_{\mathrm{bl}}(\omega)$, respectively, for the Y123 series. For $p \geqslant 0.115$ the spectra exhibit a BA mode at a finite energy instead of a Drude peak. The energy, $\omega_{0}$, and broadening, $\gamma$, of this mode increases with increasing doping, as shown in Fig. 3(g). Our results, in conjunction with the microscopic theory of Ref. [19], indicate that the bonding-antibonding splitting develops for $p$ above the critical value of $0.10 \pm 0.01$. The observed increase of $\omega_{0}$ with increasing $p$ is consistent with the assumption that the effective value of the intrabilayer hopping matrix element $t_{\perp}$ increases with doping (it has been shown in Ref. [19] that the energy of the BA mode increases with $t_{\perp}$ and the same applies to the $T_{1}$ mode corresponding to the TPM).

ARPES experiments on Y123 samples with the surface doping controlled through in situ deposition of $\mathrm{K}$ atoms reveal a collapse of the bonding and antibonding Fermi surfaces into four nodal Fermi arcs for doping levels below $p_{c}=$ $0.12 \pm 0.02[24,25]$. Our results agree within the error bars with this value and suggest that the collapse occurs likely 


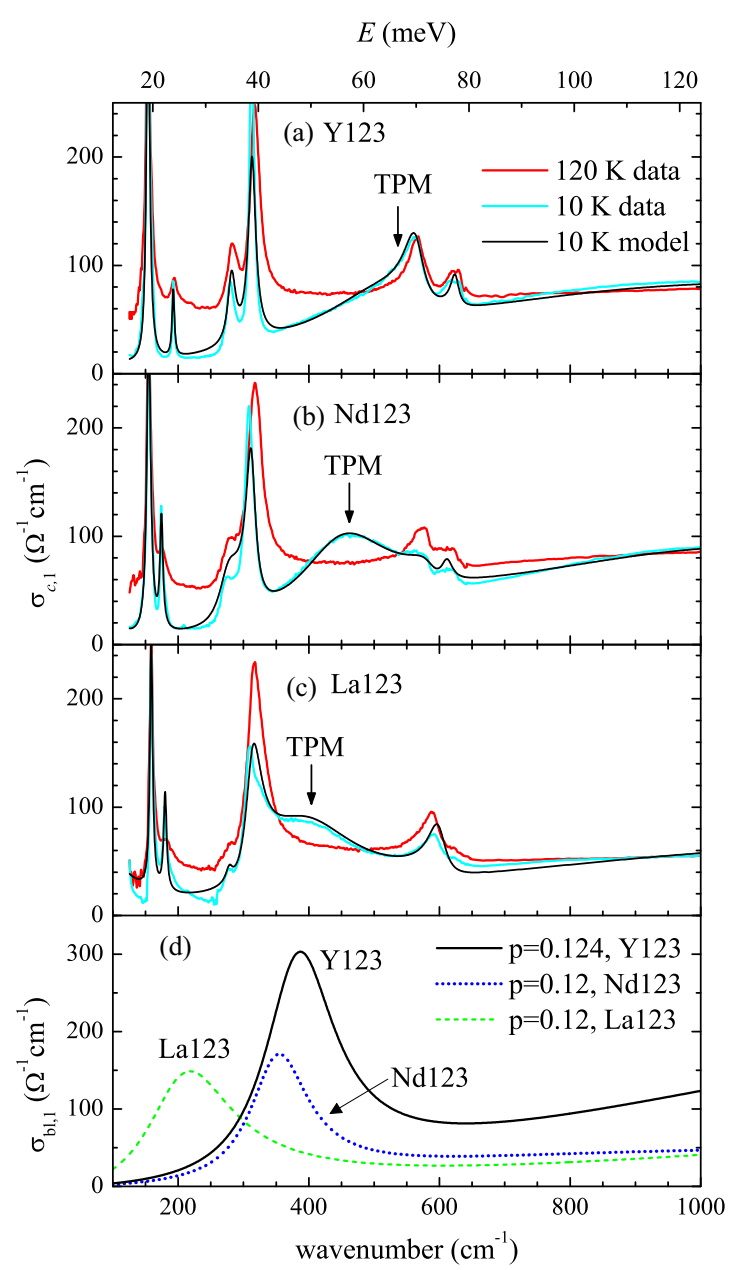

FIG. 4. Intrabilayer distance dependence of the BA mode. Panels (a), (b), and (c) show the $\sigma_{c, 1}(\omega)$ spectra at $T=10 \mathrm{~K}$ (cyan lines) and at $120 \mathrm{~K}$ (red lines) for $p \approx 0.12 \mathrm{Ln} 123$ with $\mathrm{Ln}=\mathrm{Y}, \mathrm{Nd}$, and $\mathrm{La}$, respectively. The black lines represents fits to the $10 \mathrm{~K}$ spectra obtained using the MLM model. The intrabilayer distance increases by about $8 \%$ when going from Y123 (a) through to La123 (c) [38]. Panel (d) shows the spectra of $\sigma_{\mathrm{bl}, 1}$ inferred from the MLM fitting.

within the lower part of the ARPES error-bar interval. Recall that infrared spectroscopy is a bulk-sensitive technique. As such, our results provide an important validation that the band splitting is indeed a bulk phenomenon rather than a surface effect or a result of a surface modification [25,37].

An alternative way to modify $t_{\perp}$ is via changes of the distance, $d_{\mathrm{bl}}$, between the two closely spaced $\mathrm{CuO}_{2}$ layers (the intrabilayer distance). In this case, $t_{\perp}$ is expected to decrease as $d_{\mathrm{bl}}$ increases. Experimentally, $d_{\mathrm{bl}}$ can be affected by substitutions of the $\mathrm{Y}$ ion by $\mathrm{Ln}^{3+}$ species with larger ion size, $r_{\text {ion }}$. For example, $r_{\text {ion }}(\mathrm{Y})<r_{\text {ion }}(\mathrm{Nd})<r_{\text {ion }}(\mathrm{La})$ leads to La123 having about $8 \%$ larger $d_{\mathrm{bl}}$ than Y123 [38]. Thus one might expect a lower energy BA mode for La123 as compared to Y123 of a similar doping level. We find that this is indeed the case. Figures 4(a)-4(c) shows the spectra of $\sigma_{c, 1}$ along with their respective MLM based fits (black lines) for $\mathrm{Ln} 123$ samples with $\mathrm{Ln}=\mathrm{Y}, \mathrm{Nd}$, and $\mathrm{La}$, all at a similar doping level of $p \approx 0.12$. The inferred spectra of $\sigma_{\mathrm{bl}, 1}$ shown in Fig. 4(d) are consistent with our expectations from

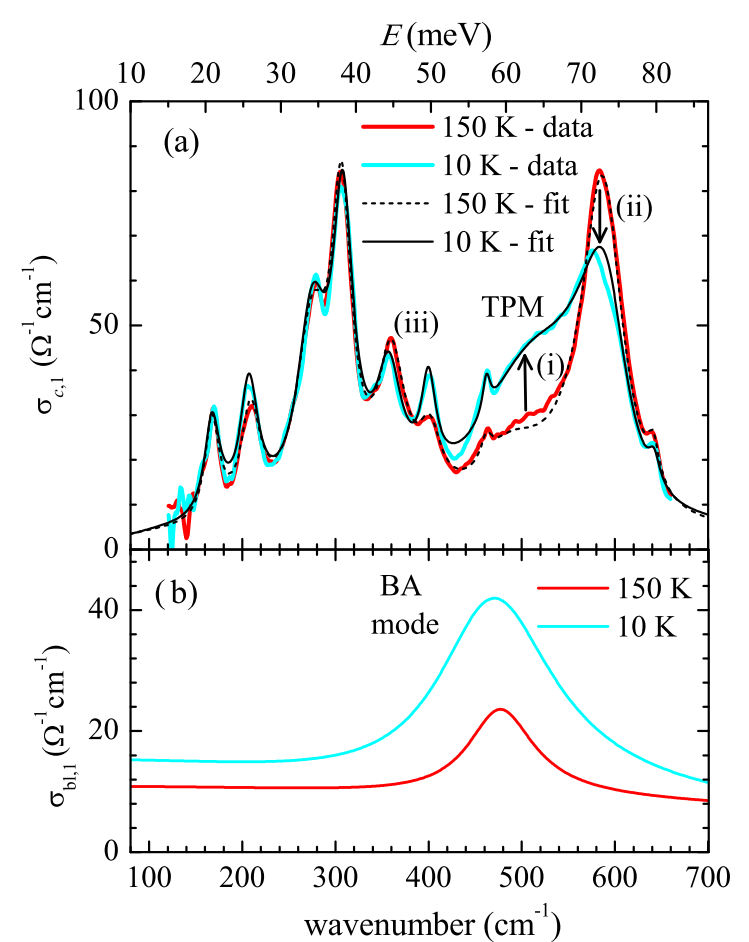

FIG. 5. (a) The real part of the $c$-axis conductivity of slightly underdoped Bi2223 with $p=0.132$ and $T_{\mathrm{c}}=102 \mathrm{~K}$. Data at $150 \mathrm{~K}$ (red line) and $10 \mathrm{~K}$ (cyan lines) and fits using the MLM for $150 \mathrm{~K}$ (dotted line) and $10 \mathrm{~K}$ (black solid line). b) The real part of $\sigma_{\mathrm{bl}}(\omega)$ corresponding to the model spectra shown in panel (a).

the arguments above- the BA mode moves to lower energy as $r_{\text {ion }}$ increases from $\mathrm{Ln}=\mathrm{Y}$ to $\mathrm{Nd}$ to $\mathrm{La}$.

\section{Bi-based cuprates}

We have also analyzed with the MLM the $c$-axis conductivity of a weakly underdoped Bi-based cuprate superconductor Bi2223 with $T_{\mathrm{c}}=102 \mathrm{~K}, p=0.132$, which exhibits a pronounced TPM feature. Figure 5(a) shows the real part of the $c$-axis conductivity of the Bi2223 single crystal. When compared with the $150 \mathrm{~K}$ data (red line), the $10 \mathrm{~K}$ data (cyan line) display several significant differences: (i) an increase in spectral weight around $500 \mathrm{~cm}^{-1}$ (a TPM feature), (ii) a softening and broadening of the $580 \mathrm{~cm}^{-1}$ phonon, and (iii) anomalous changes of the $360 \mathrm{~cm}^{-1}$ and $400 \mathrm{~cm}^{-1}$ phonons.

The features (i) and (ii) appear qualitatively similar to those for Y123 discussed above and we model them in the same way with the MLM. Next we summarize the important details of our approach. The interbilayer conductivity, $\sigma_{\text {int }}(\omega)$, has been set equal to zero since the $\mathrm{Bi}-\mathrm{O}$ "blocking layers" separating the $\mathrm{CuO}_{2}$ trilayers are almost insulating. The mode at $580 \mathrm{~cm}^{-1}$ is included as usual as an interbilayer oxygen mode (it predominantly involves in-phase $c$-axis motion of the apical oxygens [39]). The parameters of this phonon were fixed at their high-temperature values and so the anomalous temperature change of this mode is fully accounted for by the changes in $\sigma_{\mathrm{bl}}(\omega)$. The anomalous behavior of the $360 \mathrm{~cm}^{-1}$ and $400 \mathrm{~cm}^{-1}$ phonons was qualitatively interpreted in Ref. [40] in terms of their eigenvectors. For the $360 \mathrm{~cm}^{-1}$ $\left(400 \mathrm{~cm}^{-1}\right)$ mode the oxygens in the outer layers and those 
TABLE II. Values of the fitting parameters for slightly underdoped Bi2223 entering the model formulas: frequencies $\left(\omega_{0}\right)$, plasma frequencies $\left(\omega_{\mathrm{pl}}\right)$, and broadening parameters $(\gamma)$ of the oscillators obtained by fitting the $150 \mathrm{~K}$ and $10 \mathrm{~K}$ data.

\begin{tabular}{|c|c|c|}
\hline$T(\mathrm{~K})$ & 150 & 10 \\
\hline$\epsilon_{\infty}$ & 5.7 & 5.7 \\
\hline \multicolumn{3}{|c|}{$\sigma_{b l}$ oscillators $\left(\mathrm{cm}^{-1}\right)$} \\
\hline$\omega_{0}$ & 0 & 0 \\
\hline$\omega_{\mathrm{pl}}$ & 851 & 783 \\
\hline$\gamma$ & 1110 & 664 \\
\hline$\omega_{0}$ & 477 & 472 \\
\hline$\omega_{\mathrm{pl}}$ & 273 & 533 \\
\hline$\gamma$ & 86 & 150 \\
\hline \multicolumn{3}{|c|}{ High energy interbilayer oxygen phonon mode $\left(\mathrm{cm}^{-1}\right)$} \\
\hline$\omega_{0}$ & 627 & 627 \\
\hline$\omega_{\mathrm{pl}}$ & 538 & 538 \\
\hline$\gamma$ & 48 & 48 \\
\hline \multicolumn{3}{|c|}{ Mean-field phonon modes $\left(\mathrm{cm}^{-1}\right)$} \\
\hline$\omega_{0}$ & 641 & 642 \\
\hline$\omega_{\mathrm{pl}}$ & 90 & 74.6 \\
\hline$\gamma$ & 15 & 15 \\
\hline$\omega_{0}$ & 462 & 462 \\
\hline$\omega_{\mathrm{pl}}$ & 70 & 70 \\
\hline$\gamma$ & 15 & 10 \\
\hline$\omega_{0}$ & 401.2 & 399.8 \\
\hline$\omega_{\mathrm{pl}}$ & 152.1 & 155.5 \\
\hline$\gamma$ & 29.1 & 19.1 \\
\hline$\omega_{0}$ & 360.1 & 357.3 \\
\hline$\omega_{\mathrm{pl}}$ & 261.1 & 231.1 \\
\hline$\gamma$ & 35.8 & 33.6 \\
\hline$\omega_{0}$ & 306.6 & 307.9 \\
\hline$\omega_{\mathrm{pl}}$ & 309.6 & 310.3 \\
\hline$\gamma$ & 25.3 & 26.1 \\
\hline$\omega_{0}$ & 275.1 & 275.2 \\
\hline$\omega_{\mathrm{pl}}$ & 326.2 & 320.3 \\
\hline$\gamma$ & 43.1 & 40.1 \\
\hline$\omega_{0}$ & 209.1 & 207.2 \\
\hline$\omega_{\mathrm{pl}}$ & 187.2 & 199.8 \\
\hline$\gamma$ & 25.2 & 23.6 \\
\hline$\omega_{0}$ & 167.4 & 167.6 \\
\hline$\omega_{\mathrm{pl}}$ & 160.6 & 164.4 \\
\hline$\gamma$ & 18.9 & 20.5 \\
\hline
\end{tabular}

in the central layer vibrate approximately in phase (out of phase). A simple quantitative model of the anomalies of these phonons was elaborated in Ref. [16]. However, the behavior of these phonons in a real material depends very sensitively on details of the eigenvectors and of the local fields and it is thus difficult to include these phonons in the MLM based fit in a rigourous way. We have therefore decided to treat them as responding to the mean electric field rather than to the local fields. The other phonons have been treated in the same way.

The resulting fits are shown in Fig. 5(a) for $150 \mathrm{~K}$ (dotted line) and for $10 \mathrm{~K}$ (solid black line). Values of the fitting parameters are given in Table II. We see that the model fits the data reasonably well in the critical range between 450 $650 \mathrm{~cm}^{-1}$. The corresponding spectra of $\sigma_{\mathrm{bl}, 1}$ shown in Fig. 5(b) exhibit a well defined mode near $500 \mathrm{~cm}^{-1}$ and a broad background. We do not show here the alternative fit with the Drude peak in $\sigma_{\mathrm{bl}}(\omega)$ since this model fails to reproduce the data. It appears that the features (i) and (ii) of the $10 \mathrm{~K}$ data listed above are only reasonably reproduced if the resonance in $\sigma_{\mathrm{bl}}(\omega)$ is at a finite frequency near $500 \mathrm{~cm}^{-1}$. Note that the frequency of the TPM in $\sigma_{c, 1}(\omega)$ is only slightly higher than that of the BA mode in $\sigma_{\mathrm{bl}}(\omega)$ in contrast to the case of Y123. This is because for Bi2223, the BA mode has a fairly small spectral weight compared with Y123. As discussed in Appendix, the TPM occurs close to the longitudinal optical frequency of $\sigma_{\mathrm{bl}}(\omega)$, which, due to the small spectral weight, is relatively close in this case to the transverse optical frequency.

We note that the sharp structure near $480 \mathrm{~cm}^{-1}$ in $\sigma_{\mathrm{bl}, 1}(\omega)$ at $150 \mathrm{~K}$ [see Fig. 5(b)] is probably an artifact due to the fact that the phonons at 360 and $400 \mathrm{~cm}^{-1}$ are treated as noninteracting (mean-field) in the model as discussed above. In particular, the sharp dip around $440 \mathrm{~cm}^{-1}$ in the spectra of $\sigma_{c, 1}$ might be a result of an interference of the phonons with the electronic background. The small peak at $150 \mathrm{~K}$ is there essentially to model the small belly around $500 \mathrm{~cm}^{-1}$ which may not be there without the dip at $440 \mathrm{~cm}^{-1}$.

Our modeling of the infrared response of Bi2223 thus reveals a BA mode at about $500 \mathrm{~cm}^{-1}(60 \mathrm{meV})$ for $p=$ 0.132 . This value is comparable to the normal-state magnitude of the bonding-antibonding splitting in near optimally doped Bi2212 of $\sim 100 \mathrm{meV}$ that is obtained by ARPES near the antinodes in the Brillouin zone [20,41,42]. Note that the splitting is smaller away from the antinodes, with ARPES measurements indicating a value of $\sim 15 \mathrm{meV}$ near the nodes [43], and that the energy of the BA mode of Ref. [19] derives from a weighted average of the normal state splitting over all the Brillouin zone. The situation for Bi2223 is somewhat more complicated as the inner- and outer- $\mathrm{CuO}_{2}$ layers have different values of $p$, and there are now three bands crossing the Fermi level $[23,44]$. For Bi2223, Mori et al. predict that the value of the bonding-antibonding splitting is about 1.5 times larger than that for the bilayer compound [44]. Generally, the band splitting in ARPES spectra is more pronounced for overdoped samples, where the quasiparticle lifetime is much longer, whereas in our optical conductivity spectra it is more readily identifiable in slightly-underdoped samples, where the TPM feature and phonon anomalies are more pronounced.

\section{SUMMARY}

With a phenomenological multilayer model, we have quantitatively analyzed the phonon anomalies occurring in the $c$-axis conductivity of several high-temperature cuprate superconductors for various values of the hole doping, $p$. The multilayer model introduces two local conductivities: the conductivity $\sigma_{\mathrm{bl}}(\omega)$ of the region between the closely spaced $\mathrm{CuO}_{2}$ layers (the so-called intra-bilayer region) and the local conductivity $\sigma_{\text {int }}(\omega)$ of the region separating these stacks of $\mathrm{CuO}_{2}$ layers (the so-called inter-bilayer region).

The modeling of $\mathrm{YBa}_{2} \mathrm{Cu}_{3} \mathrm{O}_{7-\delta}$ data shows that bilayer splitting is already developed for $p>0.10 \pm 0.01$, with the superconductivity related maximum in the spectra of the real part of $\sigma_{\mathrm{bl}}(\omega)$ centered at a finite energy, increasing from $30 \mathrm{meV}$ at $p=0.115$ to $55 \mathrm{meV}$ at $p=0.137$. The results are in good agreement with the theoretical study of the $c$-axis response of bilayer compounds in Ref. [19] that predicts 
a collective intrabilayer mode at a similar energy due to a strong coupling of the two adjacent $\mathrm{CuO}_{2}$ layers. This mode cannot be assigned to a simple picture of a transition between the bonding and antibonding bands - the latter transitions are instead predicted to manifest themselves at higher energies and have indeed been observed in samples with $p>0.11$ [19]. For $p=0.09$, the maximum of $\sigma_{\mathrm{bl}, 1}(\omega)$ is centered essentially at zero energy which points to a weak (Josephson) coupling. This doping dependence is in agreement with the trends deduced from angle-resolved photoemission data where the onset of a strong intrabilayer coupling (i.e., well resolved bonding and antibonding bands) occurs at $p_{c}=0.12 \pm 0.02$ [25], with our results showing that this splitting of the conduction band is indeed a bulk effect.

\section{ACKNOWLEDGMENTS}

The authors would like to thank Dr. J. Chaloupka for useful discussions on this work. This work was supported by the Schweizerische Nationalfonds (SNF) through Grant No. 200020-172611 and by the MEYS of the Czech Republic under the project CEITEC 2020 (LQ1601). B.P.P.M. acknowledges support from the Rutherford Foundation of New Zealand. Some measurements were performed at the IR beam line of the ANKA synchrotron at FZ Karlsruhe, where we acknowledge the support of Y.L. Mathis and M. Süpfle.

\section{APPENDIX: FORMULA FOR THE FREQUENCY OF THE BA MODE IN A SIMPLE CASE}

Here we derive the formula for the frequency of the BA mode in the most simple case of vanishing interbilayer conductivity and electron-phonon coupling neglected. In the absence of any phonon contribution, the formula for the $c$-axis dielectric function yielded by the solution of the complete set of equations (see Sec. 4 of Ref. [30]) reads

$$
\frac{1}{\epsilon(\omega)}=\frac{z_{\mathrm{bl}}}{\epsilon_{\mathrm{bl}}(\omega)}+\frac{z_{\mathrm{int}}}{\epsilon_{\mathrm{int}}(\omega)},
$$

where $z_{\mathrm{bl}}=d_{\mathrm{bl}} /\left(d_{\mathrm{bl}}+d_{\mathrm{int}}\right)$ and $z_{\mathrm{int}}=d_{\mathrm{int}} /\left(d_{\mathrm{bl}}+d_{\mathrm{int}}\right)$ are the volume fractions of the bilayer and interbilayer region, respectively. Functions $\epsilon_{\mathrm{bl}}(\omega)=\epsilon_{\infty}+\chi_{\mathrm{bl}}(\omega)$ and $\epsilon_{\mathrm{int}}(\omega)=\epsilon_{\infty}+$ $\chi_{\text {int }}(\omega)$ can be viewed as the dielectric functions of the intra- and interbilayer region, respectively. Since the complex impedance is inversely proportional to the dielectric function, Eq. (A1) can be viewed as the formula for the total impedance of the set of the intrabilayer and interbilayer impedances in series, which was pointed out in the early paper by van der Marel and A. Tsvetkov [17]. If we assume $\chi_{\mathrm{bl}}(\omega)$ in the form of an undamped Lorentzian resonance at frequency $\omega_{\mathrm{BA}}$,

$$
\chi_{\mathrm{bl}}(\omega)=\frac{\omega_{\mathrm{bl}}^{2}}{\omega_{\mathrm{BA}}^{2}-\omega^{2}}
$$

and assume that the interbilayer region is insulating, $\chi_{\mathrm{int}}(\omega)=$ 0 , Eq. (A1) yields

where

$$
\epsilon(\omega)=\epsilon_{\infty}+\frac{S}{\omega_{\mathrm{TPM}}^{2}-\omega^{2}},
$$

$$
\omega_{\mathrm{TPM}}^{2}=z_{\mathrm{int}} \frac{\omega_{\mathrm{bl}}^{2}}{\epsilon_{\infty}}+\omega_{\mathrm{BA}}^{2}
$$

and

$$
S=z_{\mathrm{bl}} \omega_{\mathrm{bl}}^{2} .
$$

Since the geometrical factor $z_{\text {int }}$ is typically close to 1 in cuprates, the frequency of TPM (A4) is close to the screened longitudinal optical frequency of the intrabilayer dielectric function. Equation (A5) shows that the spectral weight increases with increasing number of $\mathrm{CuO}_{2}$ planes per unit cell provided $\omega_{\mathrm{bl}}$ is constant. We express the frequency of the BA mode from Eqs. (A4) and (A5) as

$$
\omega_{\mathrm{BA}}^{2}=\omega_{\mathrm{TPM}}^{2}-\frac{z_{\text {int }}}{z_{\mathrm{bl}}} \frac{S}{\epsilon_{\infty}} .
$$

We can see that in the limit of small spectral weight $S$, the frequency of the BA mode approaches that of the TPM, which is the case of $\mathrm{Bi} 2223$ analyzed in this paper. With increasing $S$, the BA mode frequency decreases, and it can be significantly lower than that of the TPM, as is the case of Y123 with $p=0.115$ analyzed in Sec. III A. For strongly underdoped Y $123 \omega_{\mathrm{BA}}$ seems to approach zero. Equation (A6) can be in principle used for a simple estimate of the BA mode frequency from experimental values of $\omega_{\mathrm{TPM}}, S$, and $\epsilon_{\infty}$, provided that the assumptions are reasonably valid, i.e., the compound is strongly anisotropic $\left(\chi_{\text {int }}(\omega) \approx 0\right)$ and the interactions with phonons are weak enough. In other cases, a fitting of the data with the full model with interacting phonons is needed.
[1] C. C. Homes, T. Timusk, R. Liang, D. A. Bonn, and W. N. Hardy, Phys. Rev. Lett. 71, 1645 (1993).

[2] J. Schützmann, S. Tajima, S. Miyamoto, Y. Sato, and R. Hauff, Phys. Rev. B 52, 13665 (1995).

[3] L. Yu, D. Munzar, A. V. Boris, P. Yordanov, J. Chaloupka, T. Wolf, C. T. Lin, B. Keimer, and C. Bernhard, Phys. Rev. Lett. 100, 177004 (2008).

[4] C. Bernhard, D. Munzar, A. Golnik, C. T. Lin, A. Wittlin, J. Humlíček, and M. Cardona, Phys. Rev. B 61, 618 (2000).

[5] T. Timusk and C. Homes, Solid State Commun. 126, 63 (2003).
[6] A. V. Pimenov, A. V. Boris, L. Yu, V. Hinkov, T. Wolf, J. L. Tallon, B. Keimer, and C. Bernhard, Phys. Rev. Lett. 94, 227003 (2005).

[7] A. Dubroka, M. Rössle, K. W. Kim, V. K. Malik, D. Munzar, D. N. Basov, A. A. Schafgans, S. J. Moon, C. T. Lin, D. Haug, V. Hinkov, B. Keimer, T. Wolf, J. G. Storey, J. L. Tallon, and C. Bernhard, Phys. Rev. Lett. 106, 047006 (2011).

[8] E. Uykur, K. Tanaka, T. Masui, S. Miyasaka, and S. Tajima, Phys. Rev. Lett. 112, 127003 (2014).

[9] C. Homes, T. Timusk, D. Bonn, R. Liang, and W. Hardy, Physica C (Amsterdam) 254, 265 (1995). 
[10] C. Homes, T. Timusk, D. Bonn, R. Liang, and W. Hardy, Can. J. Phys. 73, 663 (1995).

[11] W. Hu, S. Kaiser, D. Nicoletti, C. R. Hunt, I. Gierz, M. C. Hoffmann, M. Le Tacon, T. Loew, B. Keimer, and A. Cavalleri, Nat. Mater. 13, 705 (2014).

[12] C. Giannetti, M. Capone, D. Fausti, M. Fabrizio, F. Parmigiani, and D. Mihailovic, Adv. Phys. 65, 58 (2016).

[13] W. Hu, D. Nicoletti, A. V. Boris, B. Keimer, and A. Cavalleri, Phys. Rev. B 95, 104508 (2017).

[14] D. Munzar, C. Bernhard, A. Golnik, J. Humlíček, and M. Cardona, Solid State Commun. 112, 365 (1999).

[15] V. Železný, S. Tajima, D. Munzar, T. Motohashi, J. Shimoyama, and K. Kishio, Phys. Rev. B 63, 060502 (2001).

[16] A. Dubroka and D. Munzar, Physica C (Amsterdam) 405, 133 (2004).

[17] D. van der Marel and A. Tsvetkov, Czech. J. Phys. 46, 3165 (1996).

[18] S. V. Dordevic, E. J. Singley, J. H. Kim, M. B. Maple, S. Komiya, S. Ono, Y. Ando, T. Rõõm, R. Liang, D. A. Bonn, W. N. Hardy, J. P. Carbotte, C. C. Homes, M. Strongin, and D. N. Basov, Phys. Rev. B 69, 094511 (2004).

[19] J. Chaloupka, C. Bernhard, and D. Munzar, Phys. Rev. B 79, 184513 (2009).

[20] Y.-D. Chuang, A. D. Gromko, A. Fedorov, Y. Aiura, K. Oka, Y. Ando, H. Eisaki, S. I. Uchida, and D. S. Dessau, Phys. Rev. Lett. 87, 117002 (2001).

[21] D. L. Feng, N. P. Armitage, D. H. Lu, A. Damascelli, J. P. Hu, P. Bogdanov, A. Lanzara, F. Ronning, K. M. Shen, H. Eisaki, C. Kim, Z.-X. Shen, J.-i. Shimoyama, and K. Kishio, Phys. Rev. Lett. 86, 5550 (2001).

[22] A. Damascelli, Z. Hussain, and Z.-X. Shen, Rev. Mod. Phys. 75, 473 (2003).

[23] S. Ideta, K. Takashima, M. Hashimoto, T. Yoshida, A. Fujimori, H. Anzai, T. Fujita, Y. Nakashima, A. Ino, M. Arita, H. Namatame, M. Taniguchi, K. Ono, M. Kubota, D. Lu, Z.-X. Shen, K. Kojima, and S. Uchida, Physica C (Amsterdam) 470, 14 (2010).

[24] M. Hossain, J. Mottershead, D. Fournier, A. Bostwick, J. McChesney, E. Rotenberg, R. Liang, W. Hardy, G. Sawatzky, I. Elfimov, D. Bonn, and A. Damascelli, Nat. Phys. 4, 527 (2008).

[25] D. Fournier, G. Levy, Y. Pennec, J. McChesney, A. Bostwick, E. Rotenberg, R. Liang, W. Hardy, D. Bonn, I. Elfimov, and A. Damascelli, Nat. Phys. 6, 905 (2010).

[26] S. V. Borisenko, A. A. Kordyuk, V. Zabolotnyy, J. Geck, D. Inosov, A. Koitzsch, J. Fink, M. Knupfer, B. Büchner, V.
Hinkov, C. T. Lin, B. Keimer, T. Wolf, S. G. Chiuzbăian, L. Patthey, and R. Follath, Phys. Rev. Lett. 96, 117004 (2006).

[27] S. Schlachter, W. Fietz, K. Grube, T. Wolf, B. Obst, P. Schweiss, and M. Kläser, Physica C (Amsterdam) 328, 1 (1999).

[28] R. Azzam, N. Bashara, and S. Ballard, Ellipsometry and Polarized Light (North Holland, New York, 1977).

[29] C. Bernhard, J. Humlíček, and B. Keimer, Thin Solid Films 455, 143 (2004).

[30] A. Dubroka, L. Yu, D. Munzar, K. Kim, M. Rössle, V. Malik, C. Lin, B. Keimer, T. Wolf, and C. Bernhard, Eur. Phys. J.-Special Topics 188, 73 (2010).

[31] J. Vašátko and D. Munzar, Phys. Rev. B 86, 014512 (2012).

[32] M. Grüninger, D. van der Marel, A. Damascelli, A. Erb, T. Nunner, and T. Kopp, Phys. Rev. B 62, 12422 (2000).

[33] J. R. Schrieffer, Theory of Superconductivity (Addison-Wesley, Boston, 1988).

[34] N. Bogoljubov, V. V. Tolmachov, and D. Širkov, Fortschr. Phys. 6, 605 (1958).

[35] P. W. Anderson, Phys. Rev. 110, 827 (1958).

[36] Y. Hirata, K. M. Kojima, M. Ishikado, S. Uchida, A. Iyo, H. Eisaki, and S. Tajima, Phys. Rev. B 85, 054501 (2012).

[37] A. D. Palczewski, T. Kondo, J. S. Wen, G. Z. J. Xu, G. Gu, and A. Kaminski, Phys. Rev. B 81, 104521 (2010).

[38] M. Guillaume, P. Allenspach, W. Henggeler, J. Mesot, B. Roessli, U. Staub, P. Fischer, A. Furrer, and V. Trounov, J. Phys.: Condens. Matter 6, 7963 (1994).

[39] N. N. Kovaleva, A. V. Boris, T. Holden, C. Ulrich, B. Liang, C. T. Lin, B. Keimer, C. Bernhard, J. L. Tallon, D. Munzar, and A. M. Stoneham, Phys. Rev. B 69, 054511 (2004).

[40] A. V. Boris, D. Munzar, N. N. Kovaleva, B. Liang, C. T. Lin, A. Dubroka, A. V. Pimenov, T. Holden, B. Keimer, Y.-L. Mathis, and C. Bernhard, Phys. Rev. Lett. 89, 277001 (2002).

[41] A. Kaminski, S. Rosenkranz, H. M. Fretwell, Z. Z. Li, H. Raffy, M. Randeria, M. R. Norman, and J. C. Campuzano, Phys. Rev. Lett. 90, 207003 (2003).

[42] Y.-D. Chuang, A. D. Gromko, A. V. Fedorov, Y. Aiura, K. Oka, Y. Ando, M. Lindroos, R. S. Markiewicz, A. Bansil, and D. S. Dessau, Phys. Rev. B 69, 094515 (2004).

[43] T. Yamasaki, K. Yamazaki, A. Ino, M. Arita, H. Namatame, M. Taniguchi, A. Fujimori, Z.-X. Shen, M. Ishikado, and S. Uchida, Phys. Rev. B 75, 140513 (2007).

[44] M. Mori, T. Tohyama, and S. Maekawa, Phys. Rev. B 66, 064502 (2002).

[45] R. Henn, T. Strach, E. Schönherr, and M. Cardona, Phys. Rev. B 55, 3285 (1997). 